\title{
Statistics of heart failure and mechanical circulatory support in 2020
}

\author{
Robert E. S. Bowen, Thomas J. Graetz, Daniel A. Emmert, Michael S. Avidan \\ Department of Anesthesiology, Washington University, Saint Louis, MO, USA \\ Contributions: (I) Conception and design: All authors; (II) Administrative support: None; (III) Provision of study materials or patients: None; (IV) \\ Collection and assembly of data: RES Bowen, TJ Graetz, DA Emmert; (V) Data analysis and interpretation: None; (VI) Manuscript writing: All \\ authors; (VII) Final approval of manuscript: All authors. \\ Correspondence to: Robert E. S. Bowen. Department of Anesthesiology, Washington University, 660 S Euclid Avenue \#8054, Saint Louis, MO 63110, \\ USA. Email: r.bowen@wustl.edu.
}

\begin{abstract}
Heart failure is increasing in prevalence, with approximately 26 million patients affected worldwide. This represents a significant cause of morbidity and mortality. Statistics regarding heart failure patient age, hospitalization likelihood, and mortality differ significantly by country. Heart failure patients are typically classified by ejection fraction, with distinct phenotypes associated with reduced ejection fraction $(\mathrm{rEF})$ or preserved ejection fraction $(\mathrm{pEF})$. Heart failure has a significant financial impact related to hospitalization, medication, and procedural expenses. The costs of heart failure also extend to the reduced quality of life conferred by heart failure symptoms. Management of heart failure includes a variety of interventions, including mechanical circulatory support (MCS). MCS, including left ventricular assist devices (LVADs), right ventricular assist devices (RVADs) and extracorporeal membrane oxygenation (ECMO), has been a means of managing end stage heart failure. Given the relative scarcity of transplant organs, the utilization of MCS, particularly as a bridge to transplantation (BTT) has grown significantly. In this review, we discuss statistics related to heart failure and MCS. We evaluate how patients are classified and examine global trends and regional differences. We then address MCS therapies, the costs associated with heart failure, the impact of heart failure on patient quality of life, and data regarding morbidity and mortality.
\end{abstract}

Keywords: Heart failure; prevalence; statistics; mechanical circulatory support (MCS); left ventricular assist device (LVAD)

Submitted Jan 31, 2020. Accepted for publication May 13, 2020.

doi: $10.21037 /$ atm-20-1127

View this article at: http://dx.doi.org/10.21037/atm-20-1127

\section{Introduction}

Heart failure continues to plague the world; an estimated 26 million people have heart failure (1). Of the deaths attributed to cardiovascular disease in 2016, approximately 300,000 were estimated to be due to heart failure. Heart failure has been considered a pandemic, but primarily due to increasing prevalence (more people living with the disease), as opposed to increased incidence (more people diagnosed with the disease). In the United Kingdom (UK), prevalence increased $23 \%$ between 2002 and 2014. This increase in prevalence has been largely attributed to the improvements in medical management. In the United States of America (USA), 85.6 million adults have at least one type of cardiovascular disease, and it is estimated that $1-3 \%$ of adults (over 6.2 million) were living with heart failure in 2016 (2), with prevalence increasing with age. By 2030 , this number is predicted to increase to more than 8 million (3). Worldwide prevalence is similarly estimated to be between $1-3 \%$ in developed nations, with prevalence by country shown in Figure 1. After people are diagnosed with heart failure, the mortality risk is high-50\% die within 5 years, and $90 \%$ die within 10 years. When limited to an older population, utilizing the USA Medicare database, 


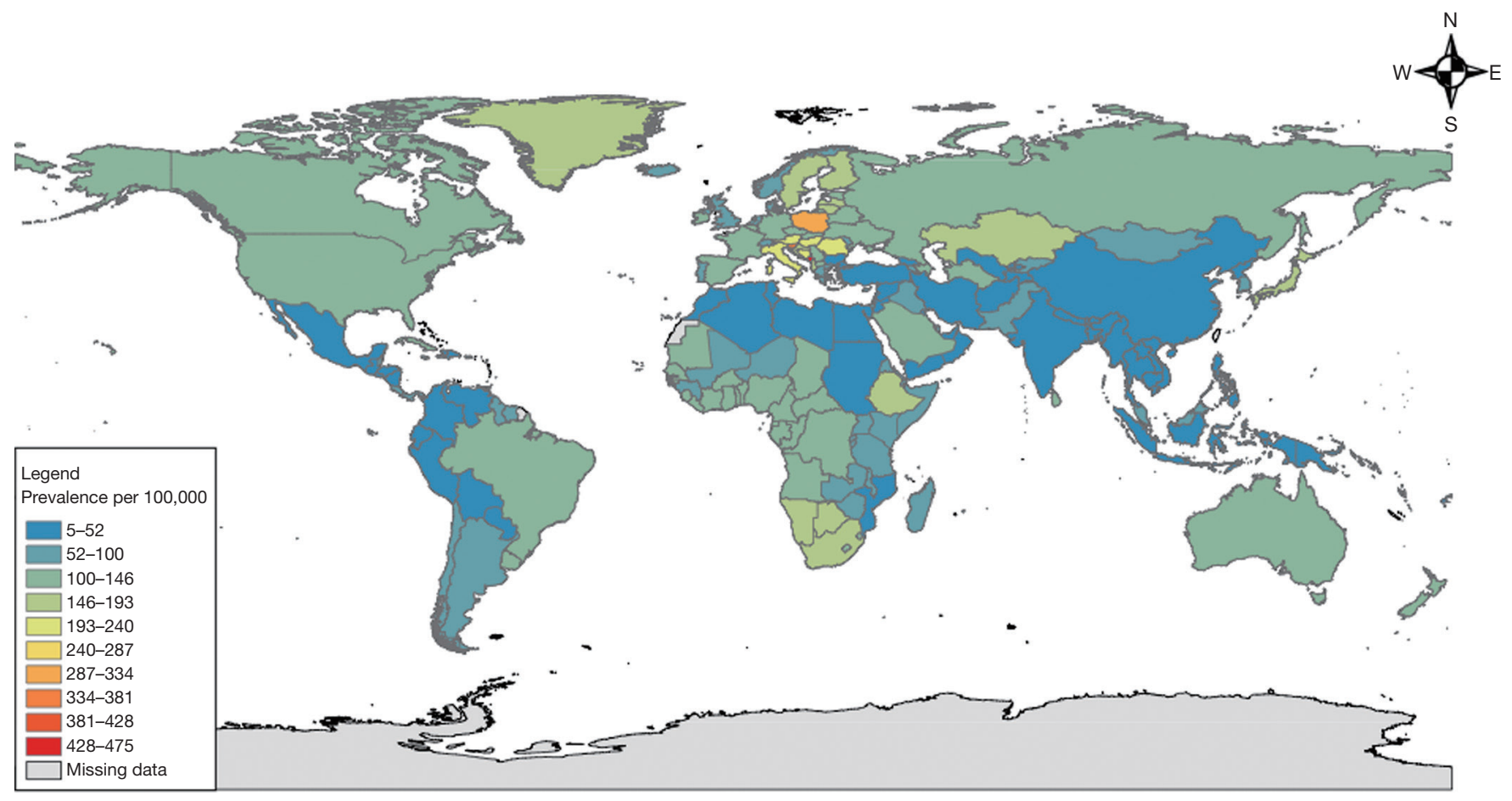

Figure 1 Age-standardized global prevalence rates of cardiomyopathy and myocarditis per 100,000, both sexes, 2017. Global Burden of Disease Study 2017. Global Burden of Disease Study 2017 (GBD 2017) Results. Seattle: Institute for Health Metrics and Evaluation (IHME), 2018. Available online: http://vizhub.healthdata.org/gbd-compare/

there is $37 \%$ mortality within 1 year, suggesting increased mortality in an older subgroup, although this may simply represent lead time bias and/or reflect unique aspects of the USA healthcare system (4).

\section{Global trends}

There is a growing body of research that attempts to evaluate the regional differences in heart failure presentation and treatment. One of the major challenges to more broadly evaluating heart failure has been a lack of data from developing nations, but recent studies have attempted to compare the heart failure patient populations in Asia and sub-Saharan Africa to Europe and North America. Compared to patients in Europe and North America, patients in Asia tended to be younger (55 vs. 67 years mean), had fewer comorbidities [including chronic obstructive pulmonary disease (COPD), despite increased smoking rates], and had less restricted functional status (5). There also tended to be different etiologies—post infectious cardiomyopathies, including rheumatic heart disease, were more common in developing nations (although decreasing in incidence relative to non-infectious etiologies, such as hypertensive or ischemic cardiomyopathies) (6), while ischemic heart disease was predominant in Europe and North America. Heart failure patients in Asia, however, had higher rates of mortality and hospitalization. Within Asian countries, however, there are significant differences. Japan and South Korea had significantly lower mortality rates than that seen in Europe and North America, and India and the Philippines had significantly lower hospitalization rates European and North American heart failure patients (7). In sub-Saharan Africa, hypertensive heart disease was similarly common, with a pooled prevalence of $39.2 \%$. Cardiomyopathy had a prevalence of $22.7 \%$, rheumatic heart disease had $13.8 \%$, and ischemic heart disease had a $7.2 \%$ prevalence. Right heart failure and pericardial disease were less common etiologies, with pulmonary tuberculosis and HIV as the leading causes, respectively. A high readmission rate (34.9\%) was also noted (6). The 
USA has noticed a downward trend in hospital admissions for heart failure, although readmission rates have increased (16.5\% any-cause) (8) for those patients who have an initial hospitalization. Rather than readmission for heart failure itself, however, comorbidities tend to drive the hospitalization (4).

\section{Patient characteristics}

Patients with heart failure are categorized as either having preserved ejection fraction ( $\mathrm{pEF}$ ), defined by a left ventricular ejection fraction greater than $50 \%$ or reduced ejection fraction ( $\mathrm{rEF}$ ) defined by an ejection fraction less than $40 \%$-these subcategories tend to differ phenotypically. Patients with pEF are more often older, female, and have higher rates of atrial fibrillation and hypertension. Patients with $\mathrm{rEF}$ are more commonly male and have a history of myocardial infarction (9). There is some thought to separate out patients with mid-range ejection fraction as well, as they tend to phenotypically mix symptoms that patients with preserved and $\mathrm{rEF}$ demonstrate. These patients with mid-range ejection fraction fall into one of three categories-patients who have improved ejection fraction (who were previously HFrEF), patients who have worsened ejection fraction (who were previously $\mathrm{HFpEF}$ ), or patients who have consistent ejection fractions in the $40-50 \%$ range. Ninety percent of patients who fell into this mid-range category are either improved or worsened ejection fraction; only $10 \%$ were stably in the $40-50 \%$ range. There have not been significant mortality differences noted between the groups, or at least, there have been inconsistencies across the literature (10).

There have been multiple attempts made to further sort patients beyond their ejection fraction. One study sorted patients with HFpEF into three phenotypic categories: young people with moderate diastolic dysfunction and normal brain natriuretic peptide levels, obese patients with diabetes, obstructive sleep apnea (OSA), and severely reduced left ventricular relaxation, and older patients with chronic kidney disease, pulmonary hypertension, and right ventricular dysfunction. Others have more simply classified patients with HFpEF by the presence or absence of specific comorbidities (2).

A recent study evaluated patient presentation and characteristics on a global scale, REPORT-HF. It found that patients commonly presented due to ischemia in Southeast Asia (25.6\%), the Western Pacific (17.1\%), the Middle East and Africa (16.2\%). Hypertension, type 2 diabetes, and chronic kidney disease were common universally $(63.6 \%$, $36.7 \%$, and $20.3 \%$ respectively). Patients in Southeast Asia, Africa and the Middle East were noted to have worse baseline functional status [New York Heart Association (NYHA) class], while patients in Europe were noted to be much more likely to have atrial arrhythmia histories compared to patients in Southeast Asia (45.7\% vs. 8.2\%). COPD was a more common comorbidity in North America than elsewhere (27\% vs. 19\%) (5).

\section{Mechanical circulatory support (MCS) in heart failure}

MCS may be offered to patients with advanced heart failure. Multiple different registries and collectives have been created to share data about patients receiving MCS. The Interagency Registry for Mechanically Assisted Circulatory Support (INTERMACS) was established in 2005. INTERMACS joined the Society of Thoracic Surgeons (STS) National Database as one of its components on January 1, 2018 (11). Additional databases include the European Registry for Patients with Mechanical Circulatory Support (EUROMACS) of the European Association for Cardio-Thoracic Surgery (EACTS) (12), the Japanese registry for Mechanically Assisted Circulatory Support (J-MACS) (13), and the UK Registry. The International Society for Heart and Lung Transplantation (ISHLT) Mechanically Assisted Circulatory Support (IMACS) has collected data since it originated in 2013. It currently collects data from STS-INTERMACS, EUROMACS, J-MACS, UK Registry, and 24 additional hospitals. The $3^{\text {rd }}$ IMACS report, with data through December 31, 2017, indicated it had collected data from over 16,000 patients from across the world (14). Data from these registries provide robust information on the MCS patient population. Additionally, long-term follow-up from prospective large trials such as MagLev Technology in Patients Undergoing Mechanical Circulatory Support Therapy with HeartMate 3 (MOMENTUM 3) (15) and Registry to Evaluate the HeartWare Left Ventricular Assist System (ReVOLVE) also provide valuable data (16). In addition to MCS, patients with heart failure may be candidates for heart transplantation. A Registry for Thoracic Organ Transplantation documents recipients of heart and heartlung transplants and is maintained by ISHLT, with over 131,000 adult heart transplant recipients through June 
Table 1 INTERMACS profile descriptions (14)

\begin{tabular}{llr}
\hline INTERMACS profile & \multicolumn{1}{c}{ Description } & LVAD implantation percentage 2013-17 \\
\hline 1 & Critical cardiogenic shock & $17 \%$ \\
2 & Progressive decline, despite intravenous inotropic support & $33 \%$ \\
3 & Stable on intravenous inotropic support & $34 \%$ \\
4 & Resting symptoms on oral therapy & $13 \%$ \\
5 & Exertion intolerant, comfortable at rest, but no activity & $2 \%$ \\
6 & Exertion limited & $1 \%$ \\
\hline
\end{tabular}

INTERMACS, the Interagency Registry for Mechanically Assisted Circulatory Support; LVAD, left ventricular assist device.

2018. It is one of the most robust of the aforementioned databases (17).

First generation left ventricular assist devices (LVADs) were refined in the 1990s (HeartMate I, HeartMateVE, and HeartMate XVE) and were pulsatile volume displacement pumps which generated forward flow. Second generation LVADs (HeartMate-II) were continuous flow, rotary pumps. They used continuous axial flow to pull blood through the pump and generate forward flow. These devices were smaller and had fewer moving parts. The third generation LVADs (HeartMate-III and HeartWare VAD) used centrifugal pumps with a spinning impeller to propel blood and produce blood flow (18). As pumps continue to become more advanced, ongoing controversy persists regarding the appropriate time to implant the devices $(11,19)$.

Categories have been developed to classify heart failure patients based on their clinical picture. The INTERMACS clinical profiles are used to describe various clinical pictures and serve as a shorthand for communication for severity of heart failure in patients that are receiving optimal medical therapy (20).

The IMACS database provides some insights into the number and types of devices over time. IMACS provides yearly data regarding centrifugal $v s$. axial devices over time 2013-2017 with 884 vs. 2,279, 1,229 vs. 2,176, 1,339 vs. 2,405, 1,371 vs. 2,064 and 1,360 vs. 1,179 respectively. Over this period, the drop in centrifugal pumps implanted in 2017 was likely due to the MOMENTUM-3 trial and these patients being ineligible for inclusion in INTERMACS dataset. Over this time-period, 16,194 LVADs have been implanted and the proportion of patients fitting INTERMACS profiles 1 through 7 has remained about the same each year as shown in Table 1.

Heart transplantation continues to evolve. This evolution is driven, in part, by changes in the donor allocation systems with the use of extended-criteria donor hearts [donation after circulatory death (DCD), ex vivo perfusion supported hearts, hepatitis C]. The expansion of the donor criteria is because the demand for organs greatly outpaces supply. Yearly transplant volume has increased over the last decade. From 2010-2018, the median recipient age was 55 years, the median donor age was 35 years, approximately $40 \%$ of donor cause of death was head trauma, and approximately $20 \%$ was stroke. The growth of pre-transplant MCS has been rapid from 2007 until present, but seems to be stabilizing at the current level of approximately $50 \%$; LVADs make up approximately $45 \%$, right ventricular assist devices (RVADs) approximately 3\%, total artificial hearts (TAHs) approximately $1 \%$ and extracorporeal membranous oxygenation (ECMOs) approximately 1\% (17). Figure 2 provides additional information over time as it relates to heart transplantation from various types of MCS. Long term mortality is similar for transplantation from MCS, other than ECMO as shown in Figure 3.

Availability of LVADs is functionally limitless, but heart transplants are limited by the donor supply. Ongoing consideration of ways to expand the donor pool is taking place. The first reported heart transplant following donation after circulatory death (DCD) took place in a pediatric series in 2008 (21). The expansion of this technique has continued as a way to expand the limited heart donor pool. Importantly, the technique of heart transplant following DCD has generated ethical concerns and controversy $(22,23)$. Most donations of organs are after brain-stem determined death, but there are a growing number of centers around the world including Australia, UK, and Belgium (24). Centers that are performing heart transplant after DCD have been having comparable short- 

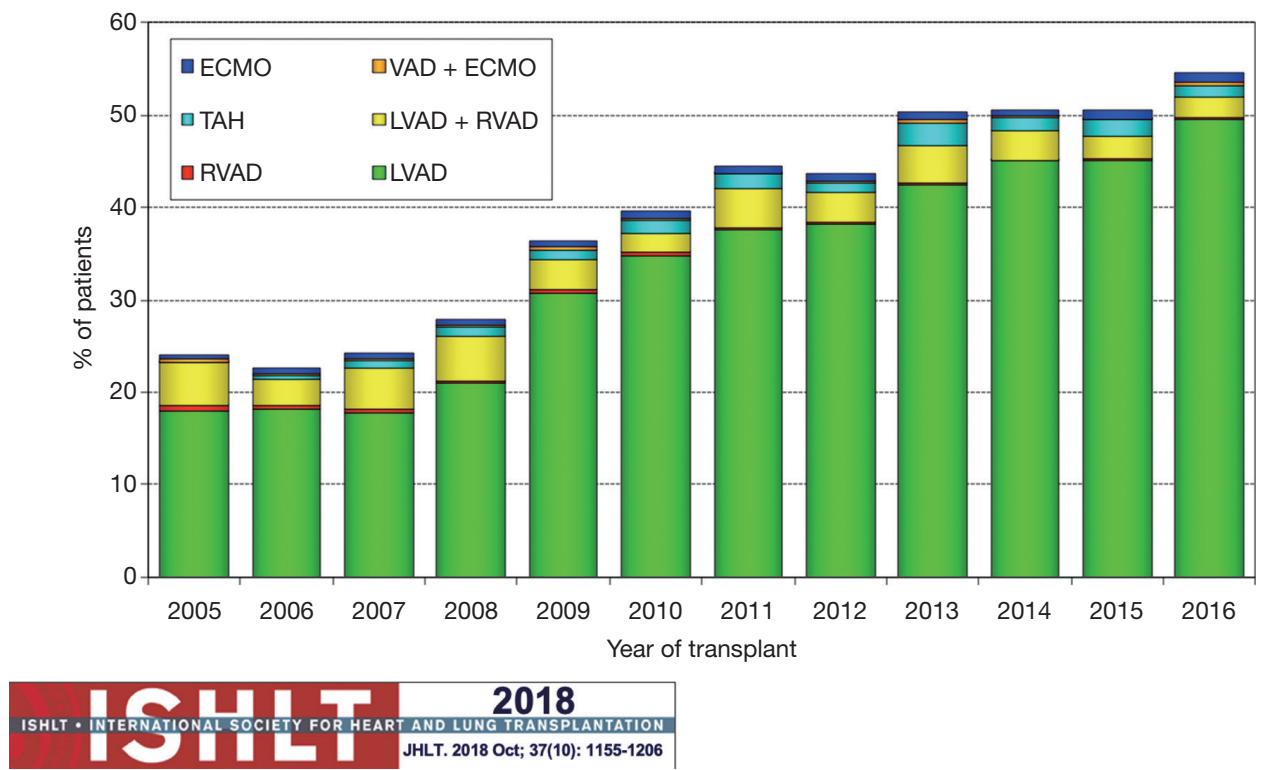

Figure 2 Adult heart transplants \% of patients bridged with MCS (LVAD, RVAD, TAH, ECMO) by year and device type (17). From: International Society for Heart and Lung Transplantation. Available online: https://ishltregistries.org/downloadables/slides/2019/heart_ adult.pptx; with permission. MCS, mechanical circulatory support; LVAD, left ventricular assist device; RVAD, right ventricular assist device; TAH, total artificial heart; ECMO, extracorporeal membrane oxygenation.

term outcomes $(25)$.

\section{Financial impact}

The costs of heart failure on a global scale are difficult to ascertain. There are numerous challenges to comparing costs across countries. Challenges cited include how the disease itself is defined, how the treatments are valued, and where the data are sourced. A study primarily evaluating North America, Europe, and Asia found that annual costs ranged (in international dollars) from $\$ 868$ to $\$ 25,532$, with an increase in cost noted as NYHA classification increased (with NYHA class IV patients comprising $70 \%$ of heart failure expenditures), increased with comorbidities (in particular diabetes mellitus), and increased toward the end of life. The country of residence has been significant, with the highest costs incurred in Germany and the USA (26).

The intersection of the increasing prevalence of heart failure with the rising cost of healthcare technology was predicted as early as 1985 by a working group at the National Heart, Lung, and Blood Institute who hypothesized that the future advent of MCS would be an expensive therapy to provide and afford (27). This prescient prediction has sparked debates and studies over the last
35 years as technological advances in advanced heart failure therapies have grown. Prior to the groundbreaking REMATCH trial, bridge to transplant (BTT) LVAD implantation was deemed reasonable because of the durability of eventual heart transplant survival and the abysmal outcomes of medical management for advanced heart failure (28). However, the dramatic mortality benefit demonstrated in the REMATCH trial, coupled with the rate-limiting availability of donor hearts, portended an increased utilization of LVAD therapy for destination therapy (DT) and a concomitant increase in related direct and indirect healthcare costs. These predictions were born out with the FDA approval of BTT therapy in 2008, and DT therapy in 2010. The direct and indirect healthcare costs of heart failure are predicted to increase dramatically with total direct and indirect costs expected at nearly $\$ 53$ billion and $\$ 17$ billion, respectively, by 2030 (29).

The early studies reporting costs and cost-effectiveness of LVADs are likely not relevant because of the significant differences in design between first and current third generation devices. However, these findings do provide a reference point for cost analysis. Most early cost analyses report daily costs or incremental costs without taking into account efficacy. A Danish study calculated cost per life- 


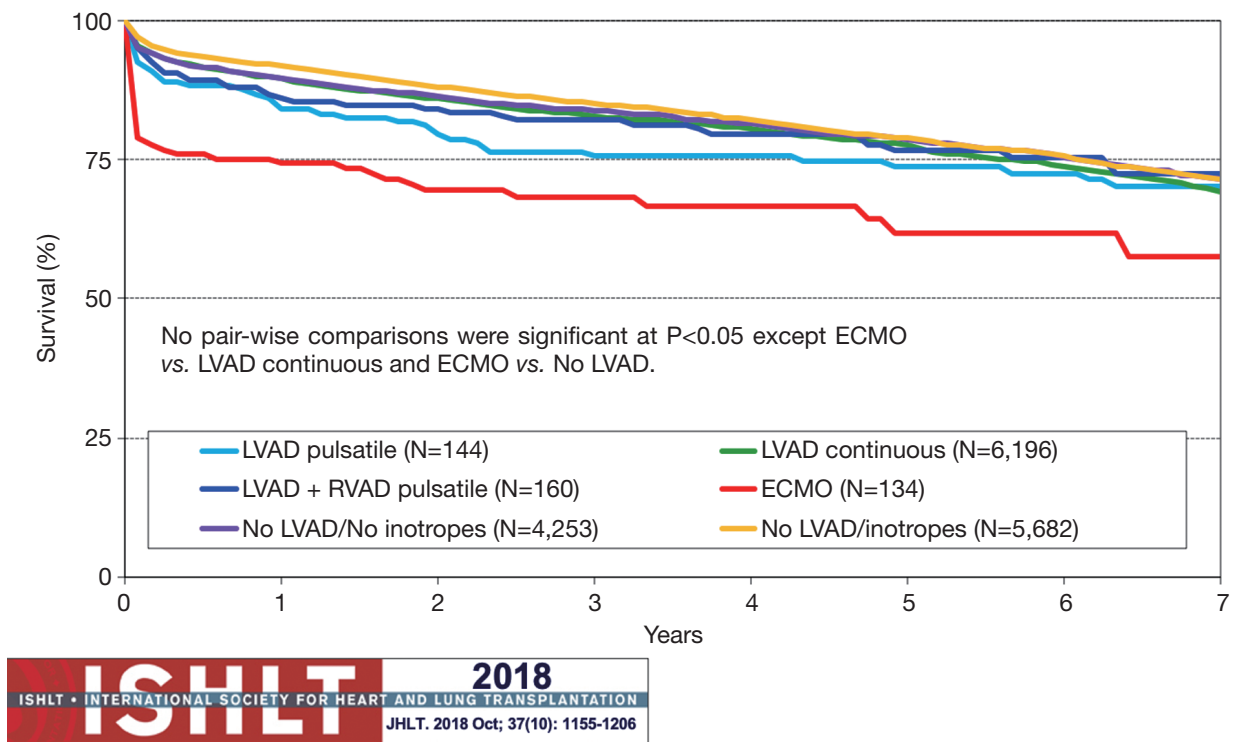

Figure 3 Adult heart transplants Kaplan-Meier survival by pre-transplant MCS use (transplants: January 2009-June 2016) (17). From: International Society for Heart and Lung Transplantation. Available online: https://ishltregistries.org/downloadables/slides/2019/heart_ adult.pptx; with permission. MCS, mechanical circulatory support; LVAD, left ventricular assist device; RVAD, right ventricular assist device; ECMO, extracorporeal membrane oxygenation.

year saved as $\$ 48,000$ (30), while a study from the UK reported the cost per quality adjusted life year (QALY) to be $\$ 78,000$ for BTT usage (31). Along the journey from early cost analysis, evidence accrued which questioned the cost effectiveness of second-generation devices. A more robust analysis in the UK of the HeartMate II for BTT demonstrated a significantly increased cost of $£ 258,922(\$ 414,275)$ per QALY compared to conventional management until heart transplantation (32). The reduced cost efficacy of the HeartMate II was presumed to be due to the improved survival of heart transplant recipients, and the significant acquisition costs of the device hardware. The cost-effectiveness of the third generation HeartWare was compared to the HeartMate II in the UK for BTT patients which demonstrated both improved efficacy and reduced costs of HeartWare device as demonstrated by an incremental cost-effective ratio (ICER) of $\$ 38,697$ over the lifetime of the patient because of the improved survival to transplantation, which incurs greater costs (33). ICER serves as a robust cost-utility analysis to compare the costs per QALY gained between interventions.

Although, no direct head-to-head comparison has been performed, the cost-effectiveness of LVAD DT has demonstrated to be less convincing in comparison to BTT therapy. A variety of cost analyses of LVAD
DT compared to medical management have reported significantly increased costs per QALY. Utilizing data obtained from the REMATCH trial, investigators reported the ICER of $\$ 198,184$ per QALY compared to medical management, which equates to a $75 \%$ reduction in ICER, from $\$ 802,702$ per QALY, for first generation, pulsatile flow device (34). USA Medicare data, likewise, has reported comparable ICER of $\$ 209,400$ per QALY; of which, a significant factor is the increase in readmission rates after LVAD implantation (35). Because of these increased costs associated with both device implantation and readmissions, more cost-conscious healthcare systems, such as Canada and the UK, have resisted the expansion of DT LVAD therapy.

\section{Quality of life impact}

Quality of life for heart failure patients is typically measured utilizing instruments such as the Kansas City Cardiomyopathy Questionnaire (KCCQ) or the Minnesota Living with Heart Failure Questionnaire (MLHFQ). These instruments utilize questions regarding activities of daily living, shortness of breath, and edema to ascertain the impact of heart failure on patient lives. Generally, scores tend to improve over time, but few medical aspects 
improve the scores. Exercise and spironolactone have demonstrated increased scores compared to control or placebo. Ultimately, the functional limitations due to heart failure (as classified by the New York Heart Association) is a significant predictor of readmission and mortality (2). Patients in Japan were scored using the Barthel index, an instrument that assesses a patient's ability to perform activities of daily living. Approximately $43.4 \%$ of patient scores worsened, which, along with low baseline scores, correlated with being discharged to a non-home location, to require a longer hospitalization, be readmitted, and suffer mortality (36).

Following LVAD implantation, there is an initial decline in quality of life indicators followed by substantial improvement. A study utilizing the INTERMACS registry and KCCQ survey data noted a baseline median score of 34.6 (scored 0-100, with 100 representing no impact on quality of life) with median scores of 14.3 at 3 months, 28.1 at 6 months, 41.1 at 9 months, at 63.8 at 1 year. There is some suggestion that there is a large improvement in quality of life following MCS utilization. These numbers may be biased as there was a significant association between 3-month KCCQ scores and mortality, but comparison of mortality across score quartiles did not differ significantly beyond this point (37).

\section{Mortality data and trends}

Considering the high mortality associated with heart failure, patient outcomes are closely studied, particular in Europe and North America. OPTIMIZE-HF, IMPACTHF, EFFECT, and the EuroHeart Failure Survey have demonstrated no significant difference in mortality or rehospitalization between patients with reduced and $\mathrm{pEF}$. OPTIMIZE-HF reported mortality rates of $37.5 \%, 35.1 \%$ and $35.6 \%$ at 1 year for patients with reduced, mid-range, and $\mathrm{pEF}$ in patients in the USA. EFFECT demonstrated similar numbers for 1-year mortality for patients with reduced and $\mathrm{pEF}(25.5 \%$ and $22.2 \%)$ in the Canadian population. The EuroHeart Failure Survey noted an estimated 1 -year mortality rate of $23.6 \%$ for acute heart failure and $6.4 \%$ for chronic heart failure. The Swedish Heart Failure Registry was utilized 12 years of data to compare mortality between patients with reduced and $\mathrm{pEF}$ and found no significant difference. One-year all-cause mortality for heart failure patients in Spain was 14\%, but found that those patients with recent hospitalization faced an increased likelihood (24\%) (38).
In other areas of the world, data are more complete. Thirty-day mortality rate in China is reported to be $5.3 \%$, while in Taiwan $3.9 \%$. In Singapore, a $2.5 \%$ mortality rate over 7 years was listed. A study of Korean patients reported $9.2 \%$ mortality at 1 year, and a study of patients in Japan reported $8.9 \%$ mortality in patients with $\mathrm{HFrEF}$ and $11.6 \%$ mortality in patients with $\mathrm{HFpEF}$ at 1 year. Numbers from South America and Australia are more similar to those reported in Europe and North America, with a respective 1 -year mortality of $24.5 \%$ and $20.5-20.7 \%$ (10).

A multicenter European study evaluating mortality and guideline adherence by physicians found that patients who saw physicians who followed guideline-based practice were $93 \%$ as likely to die as those patients who did not follow guideline-based practice over the 18 -month period of the study (39).

Data would suggest that there has been a significant decline in mortality secondary to heart failure over the past 50 years. A study from the USA demonstrated a $70 \%$ decline from 206 per 100,000 in 1968 to 62 per 100,000 in 2017. This rate, however, has not significantly changed since 2011 (40).

For patients who have undergone MCS, survival is approximately $83 \%$ at 1 year (11). The INTERMACS classification system, as previously mentioned, is the most common method of describing MCS candidates. However, more specific predictive tools have been developed for mortality and outcomes following LVAD implantation (37). The Destination Therapy Risk Score was initially utilized, but was found to be poorly predictive in continuous flow VAD patients (41). The HeartMate II Risk Score (HRMS) was designed to predict 90-day mortality in patients undergoing LVAD implantation and was defined as a function of age, international normalized ratio, albumin, creatinine, and center volume. Stratification of INTERMACS class I patients into low, mid, and high categories predicts a 2 -year mortality of $8 \%, 12 \%$, and $32 \%$, respectively, with a c-statistic of 0.7 (42). These data have had limited reproducibility, with minimal difference in mortality noted between the stratified groups when applied to other cohorts. A Bayesian model, Cardiac Outcomes Risk Assessment (CORA), has been developed that utilizes (I) intervention within the last 48 hours, (II) creatinine, (III) events experienced during the hospitalization closest to LVAD implantation, (IV) previous cardiac operations, (V) IV inotrope therapy agent, (VI) primary diagnosis, (VII) hemoglobin, (VIII) LVAD device strategy, and (IX) INTERMACS profile to predict mortality with a 
c-statistic of 0.81 for 90 -day mortality and 0.79 for 1 year mortality. The most impactful events on mortality were cardiac arrest, intubation, dialysis, extracorporeal life support, feeding tube placement, and balloon pump utilization (41). As machine learning becomes more integrated into predictive modeling in the healthcare system, there will likely be additional models to predict heart failure associated mortality (43).

\section{Conclusions}

In summary, the increased prevalence of heart failure has prompted increasing interest in both the medical management and advanced therapies because of significant effect on quality of life, mortality, and the economic burden imposed. Continued international cooperation in registry data collection and well-designed clinical outcomes trial will further identify the best timing of therapies. The tremendous growth in mechanical support for heart failure has perhaps over-shadowed medical management. However, the future of genomic-centered, patient-specific therapies may balance the treatment options available to patients.

\section{Acknowledgments}

Funding: None.

\section{Footnote}

Provenance and Peer Review: This article was commissioned by the Guest Editor (Kamen Valchanov) for the series "Perioperative Management of Patients with undergoing Mechanical Circulatory Support" published in Annals of Translational Medicine. The article was sent for external peer review organized by the Guest Editor and the editorial office.

Conflicts of Interest: All authors have completed the ICMJE uniform disclosure form (available at http://dx.doi. org/10.21037/atm-20-1127). The series "Perioperative Management of Patients with undergoing Mechanical Circulatory Support" was commissioned by the editorial office without any funding or sponsorship. The authors have no other conflicts of interest to declare.

Ethical Statement: The authors are accountable for all aspects of the work in ensuring that questions related to the accuracy or integrity of any part of the work are appropriately investigated and resolved.

Open Access Statement: This is an Open Access article distributed in accordance with the Creative Commons Attribution-NonCommercial-NoDerivs 4.0 International License (CC BY-NC-ND 4.0), which permits the noncommercial replication and distribution of the article with the strict proviso that no changes or edits are made and the original work is properly cited (including links to both the formal publication through the relevant DOI and the license). See: https://creativecommons.org/licenses/by-nc-nd/4.0/.

\section{References}

1. Ambrosy AP, Fonarow GC, Butler J, et al. The global health and economic burden of hospitalizations for heart failure: lessons learned from hospitalized heart failure registries. J Am Coll Cardiol 2014;63:1123-33.

2. Dunlay SM, Roger VL, Redfield MM. Epidemiology of heart failure with preserved ejection fraction. Nat Rev Cardiol 2017;14:591-602.

3. Khan SS, Ning H, Shah SJ, et al. 10-year risk equations for incident heart failure in the general population. J Am Coll Cardiol 2019;73:2388-97.

4. Roger VL. Epidemiology of heart failure. Circ Res 2013;113:646-59.

5. Filippatos G, Angermann CE, Cleland JGF, et al. Global differences in characteristics, precipitants, and initial management of patients presenting with acute heart failure. JAMA Cardiol 2020. [Epub ahead of print].

6. Agbor VN, Essouma M, Ntusi NAB, et al. Heart failure in sub-Saharan Africa: A contemporaneous systematic review and meta-analysis. Int J Cardiol 2018;257:207-15.

7. Dewan P, Jhund PS, Shen L, et al. Heart failure with reduced ejection fraction: comparison of patient characteristics and clinical outcomes within Asia and between Asia, Europe and the Americas. Eur J Heart Fail 2019;21:577-87.

8. Heidenreich PA, Sahay A, Kapoor JR, et al. Divergent trends in survival and readmission following a hospitalization for heart failure in the Veterans Affairs health care system 2002 to 2006. J Am Coll Cardiol 2010;56:362-8.

9. Choi HM, Park MS, Youn JC. Update on heart failure management and future directions. Korean J Intern Med 2019;34:11-43.

10. Savarese G, Lund LH. Global public health burden of heart failure. Card Fail Rev 2017;3:7-11. 
11. Kormos RL, Cowger J, Pagani FD, et al. The Society of Thoracic Surgeons Intermacs database annual report: evolving indications, outcomes, and scientific partnerships. J Heart Lung Transplant 2019;38:114-26.

12. de By TMMH, Mohacsi P, Gahl B, et al. The European Registry for Patients with Mechanical Circulatory Support (EUROMACS) of the European Association for Cardio-Thoracic Surgery (EACTS): second report. Eur J Cardiothorac Surg 2018;53:309-16.

13. Nakatani T, Sase K, Oshiyama H, et al. Japanese registry for mechanically assisted circulatory support: first report. J Heart Lung Transplant 2017;36:1087-96.

14. Goldstein DJ, Meyns B, Xie R, et al. Third annual report from the ISHLT mechanically assisted circulatory support registry: a comparison of centrifugal and axial continuousflow left ventricular assist devices. J Heart Lung Transplant 2019;38:352-63.

15. Mehra MR, Uriel N, Naka Y, et al. A fully magnetically levitated left ventricular assist device - final report. N Engl J Med 2019;380:1618-27.

16. Schmitto JD, Zimpfer D, Fiane AE, et al. Long-term support of patients receiving a left ventricular assist device for advanced heart failure: a follow-up analysis of the Registry to Evaluate the HeartWare Left Ventricular Assist System. Eur J Cardiothorac Surg 2016;50:834-8.

17. Khush KK, Cherikh WS, Chambers DC, et al. The International Thoracic Organ Transplant Registry of the International Society for Heart and Lung Transplantation: thirty-sixth adult heart transplantation report - 2019; focus theme: Donor and recipient size match. J Heart Lung Transplant 2019;38:1056-66.

18. Levine A, Gass A. Third-generation LVADs: has anything changed? Cardiol Rev 2019;27:293-301.

19. Cerier E, Lampert BC, Kilic A, et al. To ventricular assist devices or not: When is implantation of a ventricular assist device appropriate in advanced ambulatory heart failure? World J Cardiol 2016;8:695-702.

20. Stevenson LW, Pagani FD, Young JB, et al. INTERMACS profiles of advanced heart failure: the current picture. J Heart Lung Transplant 2009;28:535-41.

21. Boucek MM, Mashburn C, Dunn SM, et al. Pediatric heart transplantation after declaration of cardiocirculatory death. N Engl J Med 2008;359:709-14.

22. Macdonald P, Dhital K. Heart transplantation from donation-after-circulatory-death (DCD) donors: Back to the future-Evolving trends in heart transplantation from DCD donors. J Heart Lung Transplant 2019;38:599-600.

23. Veatch RM. Controversies in defining death: a case for choice. Theor Med Bioeth 2019;40:381-401.

24. Pettit SJ Petrie MC. Transplantation of hearts donated after circulatory-determined death. Circ Heart Fail 2019;12:e05991.

25. Messer S, Page A, Axell R, et al. Outcome after heart transplantation from donation after circulatory-determined death donors. J Heart Lung Transplant 2017;36:1311-8.

26. Lesyuk W, Kriza C, Kolominsky-Rabas P. Cost-of-illness studies in heart failure: a systematic review 2004-2016. BMC Cardiovasc Disord 2018;18:74.

27. Van Citters RL, Bauer CB, Christopherson LK, et al. Artificial heart and assist devices: directions, needs, costs, societal and ethical issues. Artif Organs 1985;9:375-415.

28. Goldstein DJ, Oz MC, Rose EA. Implantable left ventricular assist devices. N Engl J Med 1998;339:1522-33.

29. Heidenreich PA, Albert NM, Allen LA, et al. Forecasting the impact of heart failure in the United States: a policy statement from the American Heart Association. Circ Heart Fail 2013;6:606-19.

30. Kolbye A, Sander Jensen KM, Aldershvile J, et al. Economic aspects of implementation of the mechanical heart, "HeartMate". Ugeskr Laeger 2000;162:3722-5.

31. Clegg AJ, Scott DA, Loveman E, et al. The clinical and cost-effectiveness of left ventricular assist devices for endstage heart failure: a systematic review and economic evaluation. Health Technol Assess 2005;9:1-132, iii-iv.

32. Moreno SG, Novielli N, Cooper NJ. Cost-effectiveness of the implantable HeartMate II left ventricular assist device for patients awaiting heart transplantation. J Heart Lung Transplant 2012;31:450-8.

33. Pulikottil-Jacob R, Suri G, Connock M, et al. Comparative cost-effectiveness of the HeartWare versus HeartMate II left ventricular assist devices used in the United Kingdom National Health Service bridge-to-transplant program for patients with heart failure. J Heart Lung Transplant 2014;33:350-8.

34. Rogers JG, Bostic RR, Tong KB, et al. Cost-effectiveness analysis of continuous-flow left ventricular assist devices as destination therapy. Circ Heart Fail 2012;5:10-6.

35. Baras Shreibati J, Goldhaber-Fiebert J, Banerjee D, et al. Cost-effectiveness of left ventricular assist devices in ambulatory patients with advanced heart failure. JACC Heart Fail 2017;5:110-9.

36. Uemura Y, Shibata R, Takemoto K, et al. Prognostic impact of the preservation of activities of daily living on post-discharge outcomes in patients with acute heart failure. Circ J 2018;82:2793-9.

37. Flint KM, Spertus JA, Tang F, et al. Association of global 


\section{Page 10 of 10}

and disease-specific health status with outcomes following continuous-flow left ventricular assist device implantation. BMC Cardiovasc Disord 2017;17:78.

38. Farré N, Vela E, Clèries M, et al. Real world heart failure epidemiology and outcome: a population-based analysis of 88,195 patients. PLoS One 2017;12:e0172745.

39. Komajda M, Schope J, Wagenpfeil S, et al. Physicians' guideline adherence is associated with long-term heart failure mortality in outpatients with heart failure with reduced ejection fraction: the QUALIFY international registry. Eur J Heart Fail 2019;21:921-9.

40. Ritchey MD, Wall HK, George MG, et al. US trends in premature heart disease mortality over the past 50 years: where do we go from here? Trends Cardiovasc Med 2019.

Cite this article as: Bowen RES, Graetz TJ, Emmert DA, Avidan MS. Statistics of heart failure and mechanical circulatory support in 2020. Ann Transl Med 2020;8(13):827. doi: 10.21037/ atm-20-1127

\section{Bowen et al. Statistics of heart failure and mechanical support}

[Epub ahead of print].

41. Loghmanpour NA, Kanwar MK, Druzdzel MJ, et al. A new Bayesian network-based risk stratification model for prediction of short-term and long-term LVAD mortality. ASAIO J 2015;61:313-23.

42. Adamo L, Nassif M, Tibrewala A, et al. The Heartmate Risk Score predicts morbidity and mortality in unselected left ventricular assist device recipients and risk stratifies INTERMACS class 1 patients. JACC Heart Fail 2015;3:283-90.

43. Awan SE, Bennamoun M, Sohel F, et al. Machine learningbased prediction of heart failure readmission or death: implications of choosing the right model and the right metrics. ESC Heart Fail 2019;6:428-35. 